\title{
专题：地球生物学前沿
}

论 文

\section{二叠纪-三叠纪之交重大地质突变期 微生物对环境的作用}

\author{
罗根明“，谢树成，刘邓, Thomas J ALGEO \\ 中国地质大学生物地质与环境地质国家重点实验室, 武汉 430074 \\ *E-mail: logming@gmail.com
}

收稿日期: 2013-06-03; 接受日期: 2013-12-10; 网络版发表日期: 2014-05-12

国家重点基础研究发展计划项目(编号: 2011CB808800)、国家自然科学青年基金项目(批准号: 41202240)、生物地质与环境地质国家重点 实验室自主研究课题(编号: GBL11202 和 GBL11302)、高等学校学科创新引智计划(编号: B08030)和中央高校基本科研业务费专项资金(编 号: CUG120117)资助

\begin{abstract}
摘要 生物与环境之间是相互作用和协同演化的. 目前在生物对环境变化的响应方面已 取得了许多重要的认识, 而在生物对环境作用方面的认识还非常薄弱. 本文以二叠纪-三叠 纪之交这一生物与环境的重大突变期为例, 探讨了微生物对环境的作用并指出了下一步的 重点突破方向. 类脂物生物标志化合物、C-N-S 稳定同位素地球化学和矿物学的研究表明, 硫酸盐还原微生物功能群、 $\mathrm{H}_{2} \mathrm{~S}$ 的厌氧氧化微生物功能群、产甲烷微生物功能群、甲烷的 好氧氧化微生物功能群、反硝化微生物功能群和固氮微生物功能群在二叠纪-三叠纪之交显 著繁盛. 不同微生物功能群既可以加剧环境的恶化, 也可以改善环境, 正是这些多方面的微 生物作用才使得地球环境不至于向一个方向演变, 而是处于一个能够自我调节的状态.
\end{abstract}

关键词

微生物功能群

二叠纪

三叠纪

大灭绝
达尔文的进化论学说认为, 自然选择是生物进 化的主要动力, 强调了生物对自然环境的响应. 英国 气象学家 James Lovelock 于 20 世纪 70 年代初提出了 Gaia 理论(Lovelock, 1972), 认为生物与地球本身都 是类似生命体的一个部分, 该生命体具有自我平衡 或自我调节的能力, 强调了生物并不仅仅是响应环 境的变化, 而同时对环境有着重要的反作用. Lenton 等(2004)也认为生物与环境是相互作用的, 两者共同 攀爬着地球历史的长梯. 这也是目前地球生物学这 一新兴学科所要回答的核心问题之一(谢树成等, 2006).

生物对环境的作用在前寒武纪表现得最为明显.
如产甲烷微生物功能群的代谢作用对地球早期的气 候有着重要的影响(Kasting, 2005); 又如产氧光合作 用微生物功能群(特别是蓝细菌)的作用是地球大气 由无氧变成有氧的主要原因(Blankenship, 2001). 显 生宙以来, 大量的研究主要集中在生物对环境变化 的响应方面, 如探讨后生生物的爆发和辐射以及生 物大灭绝的环境致因等方面(Webby 等, 2004; Harper, 2006; Yin 等, 2007; 张元动等, 2010), 生物对环境作 用方面的研究相对较少.

Gaia 理论认为, 地质事件的出现是地球处于不 正常阶段的表现, 通过生物集体作用, 可以调节地球 演化过程中发生的这些突发性状态, 使之恢复正常,

中文引用格式: 罗根明, 谢树成, 刘邓, 等. 2014. 二叠纪-三叠纪之交重大地质突变期微生物对环境的作用. 中国科学: 地球科学, 44: 1193-1205

英文引用格式: Luo G M, Xie S C, Liu D, et al. 2014. Microbial influences on paleoenvironmental changes during the Permian-Triassic boundary crisis. Science China: Earth Sciences, 57: 965-975, doi: 10.1007/s11430-014-4822-7 
以适合生物的生长(孙枢和王成善, 2008), 因而生物 与环境的相互作用和协同演化在一些重大地质事件 中表现得尤为突出. 二叠纪-三叠纪之交是显生宙一 个重要的地质突变期. 二叠纪末期发生了显生宙最 大的一次生物集群灭绝事件(Ward 等, 2005; Erwin, 2006; Shen 等, 2011). 虽然目前对于这次生物大灭绝 的原因还存在着争议, 但有一点共识就是生物的灭 绝是由环境的巨变所引起的, 且已提出各种各样的 假说(罗根明, 2012). 相对于大量的生物对环境变化 的响应(灭绝)的研究, 有关生物对环境的反作用研究 却很少报道(Algeo 等, 2001). 本文将着重探讨二叠纪 末重大地质突变期微生物对环境的作用.

\section{1 微生物是生物与环境相互作用的重要扮 演者}

在生物与环境的相互作用中，微生物的作用尤 其重要(殷鸿福等, 2008; Konhauser, 2007. 首先, 微 生物数量庞大. 据估计, 地球上原核生物总量就达 $(4 \sim 6) \times 10^{30}$ 个细胞, 相当于 $350 \sim 550 \mathrm{Pg}$ 碳 $\left(1 \mathrm{Pg}=10^{15} \mathrm{~g}\right)$ (Whitman 等, 1998). 在整个生物进化树上, 所有的细 菌和古菌以及部分真核生物(如原生生物, 一些真菌 等)都属于微生物. 其次, 微生物时空分布广泛. 在时 间上，占据地球历史 5/6 以上演化时间的前寒武纪基 本上都是微生物主导的世界. 在空间上, 从地球表层 到地球深部数公里的范围都有微生物的足迹(Gold, 1992; Pedersen, 1993; Parkes 等, 1994; D’Hondt 等, 2004; Lipp 等, 2008; Borgonie 等, 2011). 甚至在后生 生物难以生长的一些极端环境, 微生物也广泛存在 且代谢活跃. 再次, 微生物直接与环境相互作用, 是 联系环境和其他生物的桥梁. 在生态系统中, 微生物 向上作用于动物和植物, 向下则直接作用于自然环 境. 微生物既是生产者又是分解者, 通过能量流和物 质流直接对地质环境发生作用(谢树成等, 2011). 因 此, 微生物地质过程是当前地球生物学研究中的关 键环节，是探讨地质历史时期生物与环境协同演化 的重要突破口，同时也是研究最薄弱环节(谢树成等, 2012).

二叠纪-三叠纪之交重大转折期微生物表现出与 后生生物不一样的变化特点. 一些微生物在后生生 物灭绝后出现繁盛, 如蓝细菌等(Xie 等, 2005; Jia 等, 2012; Luo 等, 2013). 同时, 后生生物灭绝后在全球
范围内都广泛沉积一套微生物岩(Lehrmann 等, 2003; 王永标等, 2005; Baud 等, 2007), 这也表明微生物在 二叠纪-三叠纪之交生物重大转折期出现非常繁盛, 对环境可能有着重要的作用.

\section{2 微生物对环境的作用}

\section{1 硫酸盐还原微生物功能群对环境的作用}

硫酸盐还原作用是厌氧微生物一种重要的产能 代谢途径，对全球的硫和碳循环具有重要意义(Shen 等, 2001; Rabus 等, 2006; Jørgensen 和 Kasten, 2006). 最新硫同位素的研究结果表明, 硫酸盐还原微生物 功能群可能起源于太古代的早期, 是地球上最早的 微生物代谢方式之一(Shen 等, 2001; Wacey 等, 2010). 硫酸盐还原微生物功能群是一类严格的厌氧微生物 (Fareleira 等, 2003; Thauer 等, 2006). 虽然也有一些 文章报道, 硫酸盐还原微生物功能群可以在有氧环 境下生存(Dannenberg 等, 1992; Lemos 等, 2001), 但 在这种情况下，微生物仅能获取少量能量以维持生 命体征但不能生长.

该代谢过程以硫酸盐 $\left(\mathrm{SO}_{4}^{2-}\right)$ 作为电子受体, 以有 机物或者氢气作为电子供体, 反应方程式如下:

$$
\begin{aligned}
& 2\left[\mathrm{CH}_{2} \mathrm{O}\right]+\mathrm{SO}_{4}^{2-} \rightarrow 2 \mathrm{HCO}_{3}^{-}+\mathrm{H}_{2} \mathrm{~S} \\
& 4 \mathrm{H}_{2}+\mathrm{SO}_{4}^{2-}+2 \mathrm{H}^{+} \rightarrow 4 \mathrm{H}_{2} \mathrm{O}+\mathrm{H}_{2} \mathrm{~S}
\end{aligned}
$$

从上述反应式可以看出, 硫酸盐被还原过程除 了将有机质氧化之外, 还伴随有一个特征的副产物, 即 $\mathrm{H}_{2} \mathrm{~S}$. 硫酸盐还原微生物功能群对地球环境有着显 著的影响，如地球早期“铁化”(海水富 $\mathrm{Fe}^{2+}$ )海洋向“硫 化”(海洋富集 $\mathrm{H}_{2} \mathrm{~S}$ ) 的转变就是由于硫酸盐还原微生 物功能群的繁盛(Canfield, 1998), 进而影响着海水化 学和生物的组成及演化(Anbar 和 Knoll, 2002).

在微生物还原硫酸盐过程中，会发生明显的硫 同位素分馏, 轻同位素 $\left({ }^{32} \mathrm{~S}\right)$ 主要富集在产生的 $\mathrm{H}_{2} \mathrm{~S}$ 中. 随着硫酸盐还原所产生的 $\mathrm{H}_{2} \mathrm{~S}$ 与金属离子的反应而 形成硫化物，使硫离开了表生硫循环系统，残余海水 的硫酸盐逐渐富集重同位素 $\left({ }^{34} \mathrm{~S}\right)$. 许多研究表明，二 叠纪-三叠纪之交海水硫酸盐硫同位素组成(通过分 析碳酸盐岩晶格中的硫酸盐(CAS)的硫同位素组成获 知, 以下简称 $\delta^{34} \mathrm{~S}_{\mathrm{CAS}}$ ) 呈现出幕式的变化 (Kaiho 等, 2001，2006; Newton 等, 2004; Riccardi 等, 2006; Gorjan 等, 2007; Luo 等, 2010; Song 等, 2014)(图 1). 


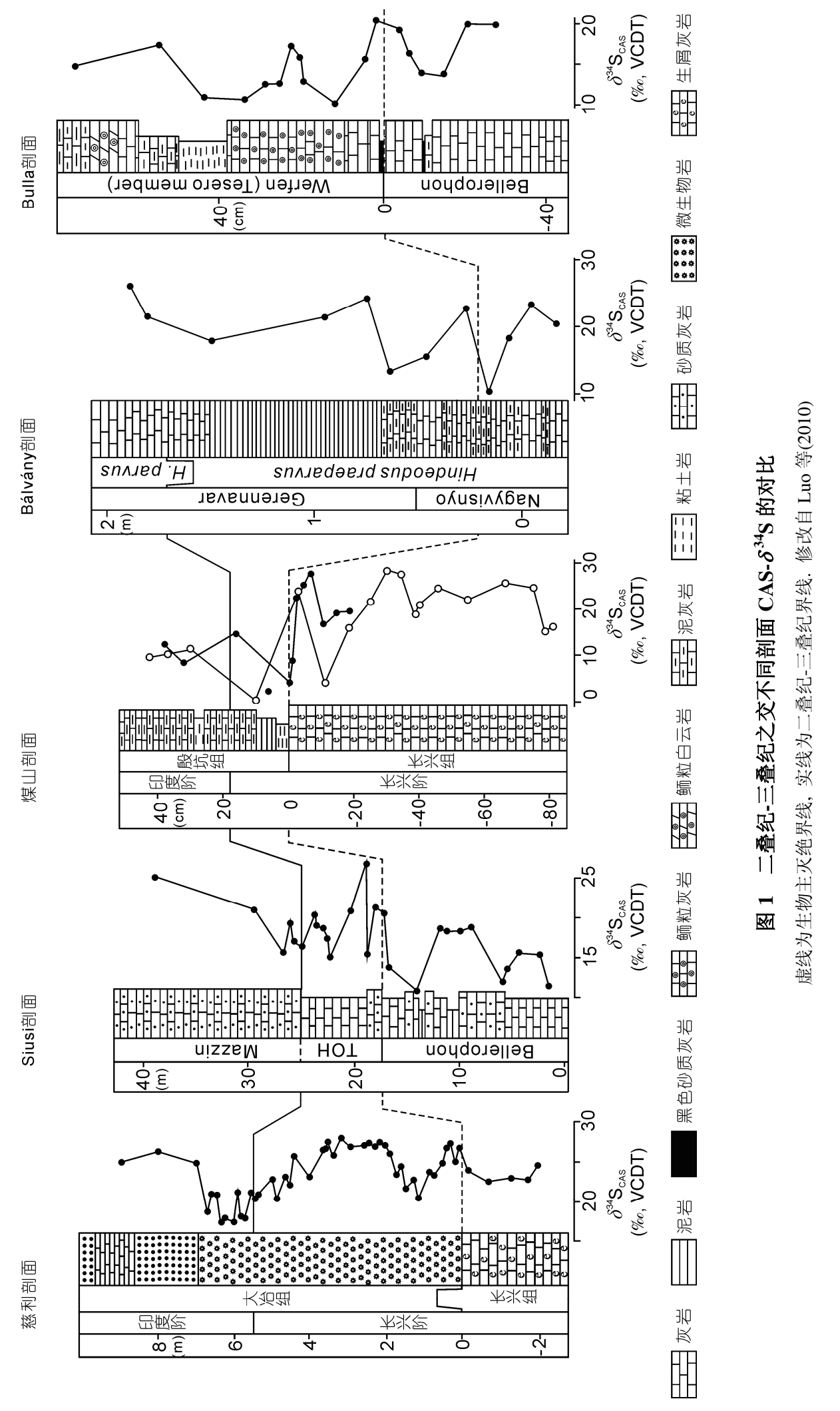


这表明二叠纪-三叠纪之交硫循环发生了显著异常. $\delta^{34} \mathrm{~S}_{\mathrm{CAS}}$ 的正向漂移, 说明当时的硫酸盐还原作用显 著加强, 进而导致 $\mathrm{H}_{2} \mathrm{~S}$ 形成通量和黄铁矿埋藏分数的 升高. 同时, 类脂物生物标志化合物的研究发现, 在 二叠纪-三叠纪之交的地层中, 出现大量来自于绿硫 细菌的生物标志化合物. 这说明 $\mathrm{H}_{2} \mathrm{~S}$ 已经进入透光层 并导致透光层缺氧, 进一步说明二叠纪-三叠纪之交 硫酸盐还原微生物功能群非常活跃. 除此之外, 二叠 纪-三叠纪之交的地层中还发现有大量的草莓状黄铁 矿(Wignall 等, 2005, 2010; Shen 等, 2007; Liao 等, 2010). 这些草莓状黄铁矿的粒径往往非常小 $(\sim 4 \mu \mathrm{m})$, 且分布比较集中, 表明其主要形成于富含 $\mathrm{H}_{2} \mathrm{~S}$ 的水 体中(Wilkin 和 Barnes, 1997). 这也表明硫酸盐还 原微生物功能群非常活跃, 其产生的 $\mathrm{H}_{2} \mathrm{~S}$ 已大量进入 水体.

$\mathrm{H}_{2} \mathrm{~S}$ 是一种具有刺激性和窒息性的无色剧毒气 体, 对多细胞生物, 特别是对后生动物是致命的. 研 究表明, 当环境中 $\mathrm{H}_{2} \mathrm{~S}$ 的浓度达到 $600 \mathrm{ppm}$ 时, 后生 动物会在一小时内死亡. 其次, $\mathrm{H}_{2} \mathrm{~S}$ 溶于水后形成氢 硫酸(一种比碳酸要强的弱酸), 从而导致水体 $\mathrm{pH}$ 的 降低, 这对钙质生物也是致命的(Hönisch 等, 2012; Knoll 等, 2007). 另一方面, 当大量的 $\mathrm{H}_{2} \mathrm{~S}$ 进入空气时, 对臭氧层有着严重的破坏作用(Kump 等, 2005), 进而 导致紫外线辐射强度的增强, 对陆地生物圈产生显 著影响.

\section{$2.2 \mathrm{H}_{2} \mathrm{~S}$ 厌氧氧化微生物功能群对环境的作用}

如上所述, 硫酸盐还原微生物功能群产生的大 量 $\mathrm{H}_{2} \mathrm{~S}$, 对绝大部分生物有重大的危害, 对大气也有 破坏作用. 然而有一些微生物能够以 $\mathrm{H}_{2} \mathrm{~S}$ 作为电子供 体 (相当于产氧光合作用中的 $\mathrm{H}_{2} \mathrm{O}$ ), 以太阳能作为能 量来源进行不产氧光合自养作用, 即 $\mathrm{H}_{2} \mathrm{~S}$ 厌氧氧化微 生物功能群, 主要包括绿硫细菌和紫硫细菌. 从这些 微生物功能群代谢所需的条件来看, 这类微生物主 要生活在富含 $\mathrm{H}_{2} \mathrm{~S}$ 的透光层. 在现代的一些封闭的分 层湖泊和局限的海湾中, 如美国纽约州的 Green Lake 和挪威的 Kyllaren Fjord, 都可以见到大量的该微生 物功能群(van Breugel 等, 2005; Zerkle 等, 2010), 并 且在某一深度可见到由于紫硫细菌大量繁盛而呈红 色的水体. 目前已知的较为可靠的最早的绿硫细菌 和紫硫细菌出现在古元古代晚期, 距今大约 16 亿年 (Brocks 等, 2005), 但对于其起源时间目前还不是很
清楚.

除此之外, 值得注意的是, 近年来研究人员们发 现, 在纳米比亚及秘鲁等地季节性 $\mathrm{H}_{2} \mathrm{~S}$ 充溢的近海海 区, 分布着一类新颖的 $\mathrm{H}_{2} \mathrm{~S}$ 厌氧氧化微生物功能群 (Lavik 等, 2009; Canfield 等, 2010a). 分子生物学工作 表明, 这类微生物功能群隶属于 $\gamma$-及 $\varepsilon$-变形菌纲, 已 被证实能利用 $\mathrm{H}_{2} \mathrm{~S}$ 作为电子供体还原硝酸盐, 同时进 行化能固碳作用(Lavik 等, 2009). 与绿硫细菌和紫硫 细菌不同的是, 其代谢能量并不是太阳能, 而是化学 能, 属于化能自养微生物功能群. 这类微生物功能群 的繁盛对环境有着重大的影响, 其大大减弱了纳米 比亚等地区高含量 $\mathrm{H}_{2} \mathrm{~S}$ 对渔业的影响(Lavik 等, 2009).

绿硫细菌和紫硫细菌有非常典型的类脂物生物 标志化合物, 且能够在地质体中很好地保存, 如芳基类异戊二烯和 isorenieratane 等(Summons 和 Powell, 1987). 这为探讨地质历史时期该微生物功能群的变 化特征提供了有效的工具. 很多学者已经在很多时 代的地层中发现了该微生物功能群的繁盛, 如白严 纪的大洋缺氧时期的地层 (Oceanic Anoxic Events, OAEs)(Sinninghe Damsté 和 Köster, 1998). 在二叠纪三叠纪之交, 到目前为止, 该类化合物已经在全球很 多地区的地层中发现, 如高纬度地区的澳大利亚、格 陵兰和挪威以及低纬地区的华南等地 (Grice 等, 2005; Hays 等, 2007; 黄咸雨等, 2007; Nabbefeld 等, 2010; Cao 等, 2009; Hays 等, 2012). 由于目前尚未发现化能 自养类型的 $\mathrm{H}_{2} \mathrm{~S}$ 厌氧氧化微生物功能群的地质生物 标志化合物, 然而不可否认这类微生物功能群可能 在二叠纪-三叠纪之交等地质历史时期的缺氧水体中 占据着重要的生态地位. 这些都表明, $\mathrm{H}_{2} \mathrm{~S}$ 厌氧氧化 微生物功能群分布非常广泛. 这些微生物大量繁盛 会消耗大量的 $\mathrm{H}_{2} \mathrm{~S}$, 进而减少了 $\mathrm{H}_{2} \mathrm{~S}$ 进入大气的通量, 减弱了因大量 $\mathrm{H}_{2} \mathrm{~S}$ 进入大气对陆地生物的和臭氧层 的破坏(Kump 等, 2005).

关于上述两类微生物功能群对 $\mathrm{H}_{2} \mathrm{~S}$ 通量变化及 环境作用的净效应, 目前还不是很确切, 还需要进一 步的定量分析. 布庸置疑的是, 硫酸盐还原微生物功 能群产生的大量 $\mathrm{H}_{2} \mathrm{~S}$ 对环境和绝大部分生物都有致 命的破坏作用, 特别是已经影响到了透光层这一绝 大部分生物生活的区间. $\mathrm{H}_{2} \mathrm{~S}$ 厌氧氧化微生物功能群 的繁盛是环境诱发的结果, 其能够缓和 $\mathrm{H}_{2} \mathrm{~S}$ 对环境的 破坏程度. $\mathrm{H}_{2} \mathrm{~S}$ 厌氧氧化微生物功能群在氧化 $\mathrm{H}_{2} \mathrm{~S}$ 的 
同时, 肯定有一部分 $\mathrm{H}_{2} \mathrm{~S}$ 逃离了该过程而进入环境. 也就是说, 从净效应来看, 硫酸盐微生物功能群的繁 盛对环境的作用更为明显.

\section{3 产甲烷微生物功能群对环境的作用}

产甲烷微生物功能群是一类严格的厌氧微生物, 属于古菌域, 可能是地球上具有最早的能量代谢形 式之一. 研究表明, 大约在 35 亿年前产甲烷微生物 功能群就已出现(Ueno 等, 2006). 产甲烷微生物功能 群的代谢主要有两大类, 一类能够以 $\mathrm{H}_{2}$ 或以乙醇为 代表的含两个或两个以上碳原子的有机物作为电子 供体, 将 $\mathrm{CO}_{2}$ 还原成 $\mathrm{CH}_{4}$, 另一类是 $\mathrm{CO}$ 或其他有机 化合物的甲基通过歧化反应的方式合成 $\mathrm{CH}_{4}$ (Canfield 等, 2004). 这类微生物功能群在调节地球早期气候方 面起着非常重要的作用(Kasting, 2005; Ueno 等, 2006). 此外, 由于 $\mathrm{CH}_{4}$ 在大气中的滞留时间很短, 可以通过 检测大气中的甲烷浓度来判断产甲烷微生物功能群 的活跃程度, 这也可能是探索火星是否有生命存在 的一个重要手段(Mumma 等, 2009).

由上述讨论可知, 产甲烷微生物功能群和硫酸 盐还原微生物功能群之间存在很强的竞争关系. 两 者都需要共同的底物, 如 $\mathrm{H}_{2}$ 和有机质(如乙酸盐)等, 进行代谢活动以获取能量(Hoehler 等, 1998; Scholten 等, 2002). 大量的研究表明, 硫酸盐在有机质的矿化 中起着非常重要的作用. 有研究表明, 现代海洋中大 约有 50\%左右的有机质是通过硫酸盐的异化还原作 用所矿化的 (Jørgensen, 1982). 这主要是因为在现代 海洋中, 硫酸盐在丰度上是仅次于氯离子的阴离子, 浓度为 $28 \mathrm{mmol} / \mathrm{L}$ 左右. 由于氧气是有机质矿化的另 一重要氧化剂, 在缺氧环境中经硫酸盐矿化的有机 质所占的比例可能更高, 所以海水硫酸盐浓度的高 低在生物地球化学循环和气候调节方面起着非常重 要的作用(Jørgensen, 1982). 早期基于盐岩中卤水液 体包裹体成分的分析认为, 二叠纪-三叠纪之交的海 水硫酸盐浓度很高, 大约为 $20 \mathrm{mmol} / \mathrm{L}$ (Horita 等, 2002; Lowenstein 等, 2005). 虽然这一浓度比现代海 洋的硫酸盐浓度略低, 但却是显生宙历史上仅次于 现代的最高值. 高的硫酸盐浓度也被认为与当时的 文石海相一致(Lowenstein 等, 2003). 然而这些数据 的分辨率很低, 并没有直接来自二叠纪-三叠纪转折 期的数据. 最近对二叠纪-三叠纪之交高分辨率的 $\delta^{34} \mathrm{~S}_{\mathrm{CAS}}$ 的分析发现, 这一重大转折期的海水硫酸盐
浓度可能非常低, 可能只有现代海洋硫酸盐浓度的 15\%左右(Luo 等, 2010). 这一结论也得到了其他方面 研究结果的支持, 如高丰度的 3-甲基蒦烷 (Cao 等, 2009)及非常低的二苯并噻吩浓度与菲浓度的比值 (DBT/P)(罗根明, 2012). 值得指出的是, 最新对鲕粒 的详细矿物学分析表明, 二叠纪-三叠纪重大转折期 可能并不是以前所认为的文石海, 而可能存在方解 石海( $\mathrm{Li}$ 等, 2013). 这也支持二叠纪-三叠纪之交海水 硫酸盐浓度很低的结论. 这些都说明从晚二叠世晚 期到二叠纪-三叠纪之交, 海水硫酸盐浓度发生了显 著的降低.

根据有机质无氧矿化的氧化还原电位对可知, 当海水硫酸盐浓度降低后, 大量的有机质可以通过 硫酸盐还原带而进入甲烷生成带(图 2). 硫酸盐浓度 的降低就为产甲烷古菌提供大量的有机质, 大大提 高了甲烷的产生通量. 另一方面, 现代海洋中产甲烷 古菌所产生的甲烷很大一部分被甲烷的厌氧氧化古 菌所消耗, 进而导致甲烷进入水体和大气的通量非 常小(Jørgensen 和 Kasten, 2006)(见 2.4). 因而, 海水 硫酸盐浓度的降低会导致甲烷的消耗通量减少, 进 而使得甲烷进入水体和大气通量的增加.

综上，二叠纪-三叠纪之交海水硫酸盐浓度的降 低, 会导致甲烷产生通量的增加, 甲烷消耗(甲烷的 厌氧氧化)的大大降低. 这会导致甲烷进入上部水体 和大气的通量显著增加. 煤山剖面所检测到的高丰 度的 3-甲基蕉烷(甲烷好氧氧化微生物功能群的生物 标志化合物(Cao 等, 2009)) 也进一步证实了甲烷进入 上部水体通量的增加. 甲烷是一种重要的温室气体 (效率相当于 $\mathrm{CO}_{2}$ 的 20 倍(Khalil, 1999)), 甲烷进入大 气通量的增加, 将会造成大气温度的显著增加(Luo 等, 2010). 这与目前牙形石氧同位素所获取的温度变 化特征相一致(Joachimski 等, 2012). 温度的升高会导 致海水溶解氧含量的减少以及大洋环流的减弱, 从 而加剧海水的缺氧程度.

\section{4 甲烷氧化微生物功能群对环境的作用}

甲烷的氧化主要由两大类微生物功能群完成, 一类是甲烷的厌氧氧化微生物功能群, 属于古菌域, 它们在无氧环境下将甲烷氧化成重碳酸根; 另一类 是甲烷的好氧氧化微生物功能群, 属于细菌域, 其代 谢活动需要氧气(Canfield 等, 2004). 愈来愈多的证据 表明, 甲烷的厌氧氧化主要是通过甲烷厌氧氧化古 


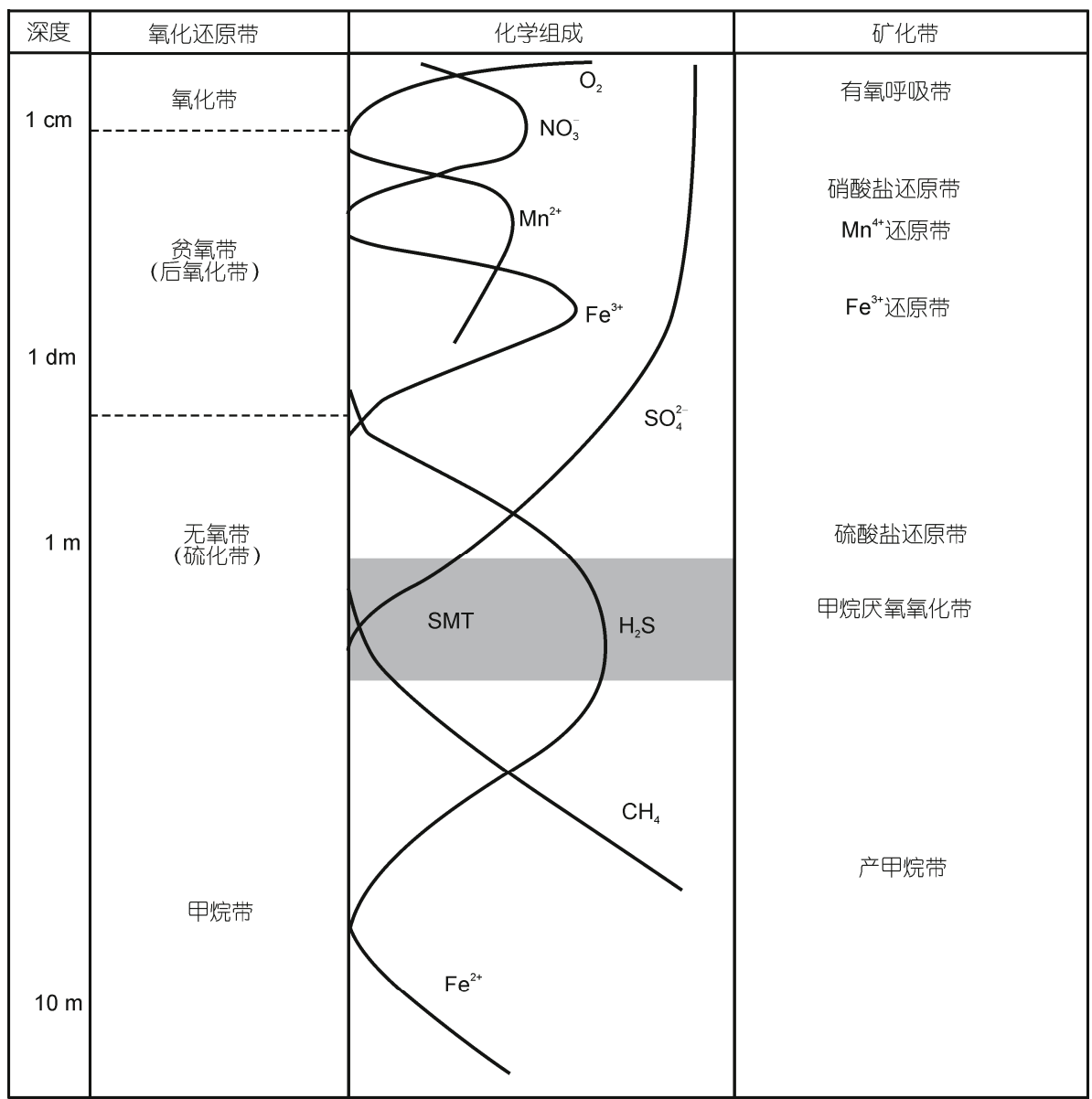

图 2 沉积物中氧含量、孔隙水的化学组成和有机质矿化带随深度的变化特征

阴影部分表示硫酸盐-甲烷过渡带(面)(SMT/SMI). 在该带中, 硫酸盐和甲烷消耗的速度最快, $\mathrm{H}_{2} \mathrm{~S}$ 的生成速度也最快. 该带之上没有 $\mathrm{CH}_{4}$, 之下则没有 $\mathrm{SO}_{4}^{2-}$. 修改自 Jørgensen 等(2006)

菌和硫酸盐还原细菌的共生作用完成的(Hinrichs 等, 1999; Orphan 等, 2001). 反应方程如下:

$$
\mathrm{CH}_{4}+\mathrm{SO}_{4}^{2-} \rightarrow \mathrm{HS}^{-}+\mathrm{HCO}_{3}^{-}+\mathrm{H}_{2} \mathrm{O}
$$

在现代海洋冷泉口, 发现了大量的甲烷厌氧氧 化微生物功能群和硫酸盐还原微生物功能群的共生 体(Blumenberg 等, 2010). 在现代海洋沉积物中, 存 在一个明显的硫酸盐-甲烷过渡带(SMT, 又称硫酸盐甲烷界面, SMI; 图 2). 在该过渡带之下, 由于产甲烷 微生物功能群的作用使得甲烷浓度很高. 该过渡带之 上由于硫酸盐浓度很高, 绝大多数甲烷都在该过渡带 被甲烷的厌氧氧化古菌通过与硫酸盐还原菌的共生作 用被厌氧氧化消耗掉. 据估计, 大约有 $90 \%$ 以上的甲 烷是通过厌氧氧化作用所消耗的(Valentine, 2002).

甲烷的好氧氧化微生物功能群是在贫氧环境下
将甲烷氧化成 $\mathrm{CO}_{2}$, 氧气是主要的电子受体(Canfield 等, 2004). 该微生物功能群主要分布于 $\gamma$-变形菌( I 型和 X 型嗜甲烷细菌)和 $\alpha$-变形菌中 ( II 型嗜甲烷细菌) (Hanson R S 和 Hanson T E, 1996). 如上所述, 在现代 海相环境中所形成的 $\mathrm{CH}_{4}$ 都被甲烷厌氧氧化微生物 功能群所消耗, 因而, 甲烷的好氧氧化微生物功能群 主要出现在陆相环境中, 如湖泊、水稻田等(Canfield 等, 2004). 据估算, 50\% 90\%左右的湿地 $\mathrm{CH}_{4}$ 被甲烷 好氧氧化微生物功能群所消耗(King 等, 1990). 综上 所述, 甲烷氧化微生物功能群在调节大气中 $\mathrm{CH}_{4}$ 浓 度及气候方面起着重要的作用.

甲烷好氧氧化微生物功能群能够产生特征的生 物标志化合物 3-甲基獲多醇，它在地质体中转化成 3-甲基蕉烷(Cvejic 等, 2000). 因此，3-甲基蕉烷能够 
很好地指示地质历史时期甲烷好氧氧化微生物功能 群的变化特征. 因为现代海洋中只有很少的甲烷能 进入沉积物-水界面处的有氧带, 从而导致现代海洋 沉积物中甲烷有氧氧化细菌的含量非常低. 这也是 为什么在现代和显生宙的海洋沉积物和原油中, 3-甲 基嚄烷和 3-甲基雚多醇的丰度都很低(Farrimond 等, 2004; Peters 等, 2005). 只有在一些低硫酸盐浓度的 湖相环境或者高通量的甲烷冷泉环境, 或者是海水 硫酸盐浓度很低的前寒武纪地层中才会出现较高丰 度的 3-甲基蕉烷或 3-甲基蕉多醇(Brocks 等, 2005; Peckmann 等, 1999; Burhan 等, 2002). Cao 等(2009)在 浙江长兴煤山剖面检测到大量的 $\mathrm{C}_{31}$ 3-甲基蒦烷, $\mathrm{C}_{31}$ 3 -甲基蕉烷指数 $\left(\mathrm{C}_{31} 3\right.$-甲基蒦烷 $/\left(\mathrm{C}_{31} 3\right.$-甲基蕉烷 $+\mathrm{C}_{30}$ 蕉烷) $\times 100$ )高达 $8 \%$. 这比显生宙的海相沉积物和石 油的典型值(1\% 2\%)要高出好几倍, 比古元古代末期 (澳大利亚的 McArthur 群的 Barney Creek 组, 约 1.64 $\mathrm{Ga}$ )所报道的 3-甲基蒦烷指数 (5.7\%)还要高(Brocks 等, 2005). 这说明, 二叠纪-三叠纪之交海洋的甲烷好氧 氧化微生物功能群非常繁盛, 也进一步支持上述二 叠纪-三叠纪之交海水硫酸盐浓度很低的结论.

如上所述, 二叠纪-三叠纪之交进入上层水体和 大气的甲烷通量显著增加, 甲烷好氧氧化微生物功 能群的繁盛将会减少进入大气的甲烷通量, 从而可 以较为有效地控制大气中甲烷的浓度, 抑制温度的 大幅升高及其后续效应. 虽然甲烷好氧氧化微生物 功能群的出现能够减少 $\mathrm{CH}_{4}$ 进入大气的通量, 但肯 定还是有一部分 $\mathrm{CH}_{4}$ 会进入大气中, 特别是甲烷好 氧氧化微生物功能群的代谢速度往往较慢. 两者之 间详细的定量关系还需下一步的工作. 二叠纪末生 物主绝灭后温度的显著升高, 也可能是由于产甲烷 微生物功能群作用的结果(Joachimski 等, 2012).

\section{5 反硝化微生物功能群对环境的作用}

氮是许多重要生化组分, 如蛋白质(包括酶)、核 酸和氨基酸等的主要组成元素. 作为生命代谢所需 的重要营养盐, 氮是海洋初级生产力的主要决定因 素之一, 影响 $\mathrm{CO}_{2}$ 的固定和大气 $\mathrm{CO}_{2}$ 浓度的调节 (Falkowski，1997). 氮循环的许多环节都是通过不同 的微生物功能群实现的(Canfield 等, 2010b).

反硝化作用指的是硝酸盐 $\left(\mathrm{NO}_{3}^{-}\right)$的异化还原作 用, 是厌氧微生物获取能量的主要途径之一(Gruber 和 Galloway, 2008). 反硝化微生物功能群的出现首先
需要环境中存在 $\mathrm{NO}_{3}^{-}$, 可以用来作为地球氧气开始 出现的一个证据(Garvin 等, 2009). 该过程以 $\mathrm{NO}_{3}^{-}$作 为电子受体进行有机质的无氧氧化, 由如下所示的 一系列的生物酶所控制(Canfield 等, 2010b).

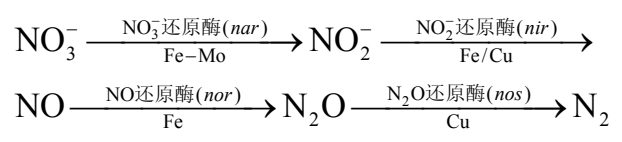

首先是硝酸盐还原酶将 $\mathrm{NO}_{3}{ }^{-}$还原成 $\mathrm{NO}_{2}{ }^{-}$(nar, nitrate reductase), 接着是亚硝酸盐还原酶(nir, nitrite reductase)将 $\mathrm{NO}_{2}{ }^{-}$还原成 $\mathrm{NO}$, 一氧化氮还原酶(nor, nitric oxide reductase) 将 $\mathrm{NO}$ 还原成 $\mathrm{N}_{2} \mathrm{O}$ 及一氧化二 氮还原酶(nos, nitrous oxide reductase) 将 $\mathrm{N}_{2} \mathrm{O}$ 还原成 $\mathrm{N}_{2}$ 而告终. 上面所示的反硝化过程表明, 这一过程 有一重要的中间产物 $\mathrm{N}_{2} \mathrm{O}$. 当反硝化作用增强时, $\mathrm{N}_{2} \mathrm{O}$ 的通量也会增加, 从而导致进入大气的 $\mathrm{N}_{2} \mathrm{O}$ 通 量的大量增加(Gruber 和 Galloway, 2008). 这一推论 也得到了地质历史时期资料的支持, 如大气 $\mathrm{N}_{2} \mathrm{O}$ 含 量在冰期时期往往较低, 而在间冰期时期则显著升 高, 这主要是因为在间冰期反硝化作用明显较冰期 强(Gruber 和 Galloway, 2008).

另一方面, 上述这些氮代谢酶都是以一些微量 金属元素(铁、钿和铜等)作为辅酶因子. 惰性气体 $\mathrm{N}_{2}$ 的形成发生在反硝化过程的最后一步, 由 $\mathrm{N}_{2} \mathrm{O}$ 还原 成 $\mathrm{N}_{2}$. 该过程所需的酶是一种同型二聚体型的金属 酶, 其重要的辅酶因子是 $\mathrm{Cu}$ (该酶的活化位置需 12 个 $\mathrm{Cu}$ 原子)(Haltia 等, 2003). 现有的模型表明, 在富 硫化氢的海洋, 海水 $\mathrm{Cu}$ 离子很快就以硫化物的形式 沉淀而离开海洋水体, 从而导致海洋 $\mathrm{Cu}^{2+}$ 的含量急 剧降低(Saito 等, 2003). Cu 含量的降低将大大减弱一 氧化二氮酶的活性, 从而导致反硝化过程停留在 $\mathrm{N}_{2} \mathrm{O}$ 的位置, 增加了 $\mathrm{N}_{2} \mathrm{O}$ 的形成通量, 并释放进入大 气中(Buick, 2007).

氮同位素的资料表明, 在二叠纪-三叠纪之交氮 循环发生了显著异常, 反硝化作用明显增强(图 3) (Algeo 等, 2007, 2012; Cao 等, 2009; Luo 等, 2011; Schoepfer 等, 2012). 这会使得 $\mathrm{N}_{2} \mathrm{O}$ 的产生通量显著 提高. 同时, 许多指标都表明, 二叠纪-三叠纪海洋的 缺氧环境迅速扩大, 出现了幕式的海洋透光层富 $\mathrm{H}_{2} \mathrm{~S}$ (Grice 等, 2005; Hays 等, 2007, 2012; Algeo 等, 2008; Cao 等, 2009). 这会使得海水中 $\mathrm{Cu}$ 含量急剧降低, 虽 然具体的变化特征还有待于进一步深入的工作. $\mathrm{Cu}$ 含量的降低会导致 $\mathrm{N}_{2} \mathrm{O}$ 通量的进一步升高. 由于 

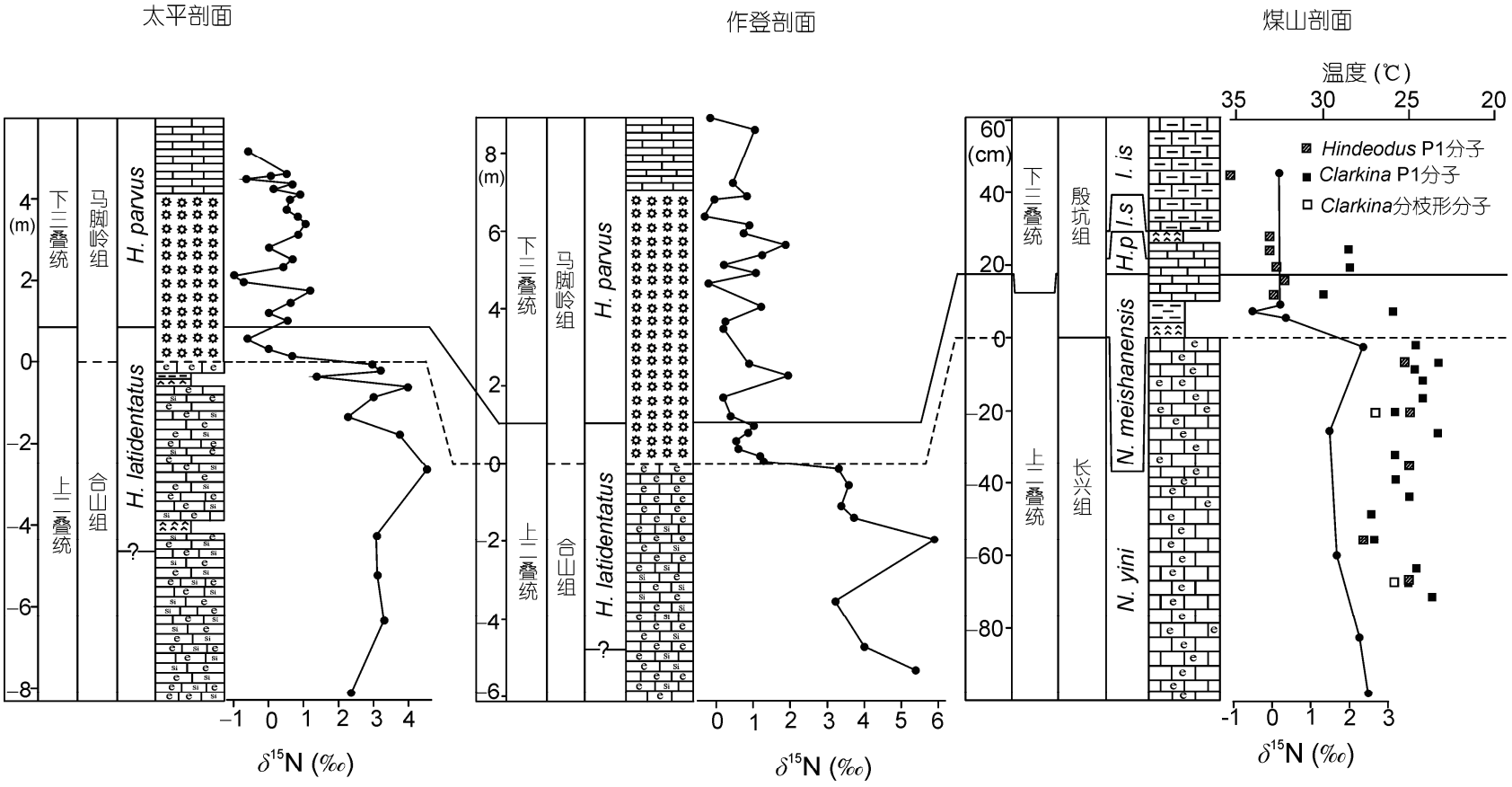

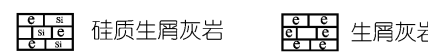

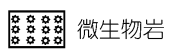

茞薄层灰岩

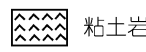

四回泥灰岩

图 3 华南地区二叠纪-三叠纪之交全岩有机质氮同位素组成 $\left(\delta^{15} \mathrm{~N}\right)$ 的变化特征和温度变化特征

太平剖面和作登剖面的 $\delta^{15} \mathrm{~N}$ 来自 Luo 等(2011); 煤山剖面的 $\delta^{15} \mathrm{~N}$ 来自 Cao 等(2009); 煤山剖面的温度为牙形石氧同位素所恢复的温度, 据 Joachimski 等(2012). 虚线为主灭绝界线; 实线为二叠纪-三叠纪界线

$\mathrm{N}_{2} \mathrm{O}$ 是一种非常强的温室气体，其效应是 $\mathrm{CO}_{2}$ 的 1000 倍, 在大气中的寿命大于 100 年(Naqvi 等, 1998). 因此, 氮循环异常将会导致二叠纪-三叠纪气温的急 剧升高. 牙形石氧同位素资料表明, 温度的升高主要 发生在主灭绝界线之上(Jochimiski 等, 2012). 这与氮 同位素指示的氮循环异常相一致, 说明反硝化微生 物功能群所释放的 $\mathrm{N}_{2} \mathrm{O}$ 可能是二叠纪-三叠纪之交温 度升高的原因之一(Joachimski 等, 2012; Romano 等, 2012; Sun 等, 2012).

\section{6 固氮微生物功能群对环境的作用}

正如上面已谈及, 生物可利用氮是影响海洋初 级生产力的重要因素(Falkowski，1997). 虽然地球表 层含有大量的氮气, 如 $78 \%$ 左右的空气都是氮气(大 约有 $4 \times 10^{21} \mathrm{~g}$ ), 然而绝大部分生物不能直接利用这些 氮气进行代谢. 这些惰性的 $\mathrm{N}_{2}$ 只有通过固氮微生物 转换成铵根之后才能被其他生物所利用. 因而, 固氮 微生物功能群的出现是生命演化史上的一个里程碑 事件, 为生物的合成代谢提供了所需的大量氮营养
盐(Falkowski, 1997; Sohm 等, 2011). 固氮微生物功能 群是一种非常古老生物, 可能在前寒武纪很早期就 已出现，对早期初级生产力的发展和演化以及早期 大气成分的变化做出了重要贡献(Beaumont 和 Robert, 1999; Navarro-Gonzalez 等, 2001).

目前为止，该功能群仅发现于原核生物中(包括 细菌和古菌)(Canfield 等, 2010b; Sohm 等, 2011)。虽 然一些真核生物也表现出固氮的能力, 如一些豆科 植物, 这主要是由与其共生的原核生物所完成的. 现 代海洋的固氮微生物主要是蓝细菌, 包括不具异形 孢的丝状蓝细菌 Trichodesmium(Capone 等, 1997)以 及单细胞蓝细菌 UCYN-A 和 Crocosphaerawatsonii (Zehr 等, 2001, 2008). 在寡营养的广海, 后两者的固 氮比率甚至比 Trichodesmium 还高(Moisander 等, 2010). 近年来，基于固氮基因(nif $\mathrm{H}$ )的研究，发现固 氮微生物的生理特征非常多样, 包括好氧自养、厌氧 自养及异养生物等(Zehr等, 1995; Severin 和 Stal, 2008, 2010), 如一些硫酸盐还原细菌.

许多研究表明，生物固氮作用所产生的氮是现 
代海洋氮库最主要的一个源, 大约每年有 $1.4 \times 10^{14} \mathrm{~g}$ 的氮是通过生物固氮作用进入海洋氮循环 (Gruber 和 Galloway, 2008). 尽管最近几年来的研究对生物固氮 通量的认识有了很大的提高, 但有些学者认为这一固 氮通量可能还是被低估了(Gruber 和 Galloway, 2008). 需要指出的是, 虽然 $\mathrm{N}_{2}$ 的还原过程是一个放热反应, 然而打开 $\mathrm{N} \equiv \mathrm{N}$ 需要很高的能量. 生物每固定 1 分子 $\mathrm{N}_{2}$ 需要 16 分子的 ATP, 因而固氮作用一般只发生在 生物可利用氮的含量很低的时候(Tyrrell, 1999).

氮同位素的资料(图 3)表明，二叠纪末期生物大 灭绝期间固氮微生物功能群非常活跃, 是生物可利 用氮的主要来源(Cao 等, 2009; Luo 等, 2011). 正是固 氮微生物功能群的繁盛补充了海洋生物代谢所需的 氮营养盐, 维持或提高了海洋初级生产力的发展, 使 得生物大灭绝后的海洋不至于成为一个“Strangelove Ocean"(Hsü 和 McKenzie, 1985). 海洋初级生产力的 维持, 吸收了大气的 $\mathrm{CO}_{2}$, 形成一个负反馈作用, 从 而使得大气 $\mathrm{CO}_{2}$ 分压不至于升得太高.

\section{3 结论及展望}

本文对二叠纪-三叠纪之交这一重大转折期生物 对环境的作用做了初步的探讨, 阐述了硫酸盐还原 微生物功能群、 $\mathrm{H}_{2} \mathrm{~S}$ 厌氧氧化微生物功能群、产甲烷 微生物功能群、甲烷好氧氧化微生物功能群、反硝化 微生物功能群和固氮微生物功能群对环境的影响. 这些微生物对环境的影响有的对生物是有利的, 如 固氮微生物功能群和 $\mathrm{H}_{2} \mathrm{~S}$ 厌氧氧化微生物功能群等, 这两者分别向海洋提供氮肥和消耗海洋中致命的 $\mathrm{H}_{2} \mathrm{~S}$ 等有毒气体. 同时, 其他微生物功能群对环境造 成的影响对其他生物是不利的, 如产甲烷微生物功
能群和反硝化微生物功能群等, 这两者分别向大气 输入大量的甲烷和 $\mathrm{N}_{2} \mathrm{O}$, 进而引起大气升温. 正是这 些微生物存在的正、负反馈作用, 使得地球环境处于 一个可调节的范围内. 二叠纪-三叠纪之交环境的频 繁波动(Yin 等, 2012)可能就是这种微生物对环境作 用的综合体现.

二叠纪末生物大灭绝后的生物复苏非常缓慢, 大约持续了 $5 \mathrm{Ma}$ 左右, 直至中三叠世早期(Chen 和 Benton, 2012), 且多次出现了错时相沉积, 如微生物 岩、巨型鲕粒等(Baud 等, 2007). 这种缓慢的复苏是 否与上述的微生物功能群对环境的作用有关, 目前 还不清楚. 已有研究表明, 整个早三叠世的海洋普遍 处于一种缺氧的状态(Song 等, 2012), 这暗示着硫酸 盐还原微生物功能群在整个早三叠世非常活跃, 虽 然其活跃程度可能也表现出阶段性的变化 (Grasby 等, 2012). 初步的研究表明, 与甲烷代谢相关的微生物 功能群在早三叠世也可能非常繁盛(Saito 等, 2013). 目前对这些微生物功能群在时间上的变化及对环境 的作用程度还不是很明确, 是下一步的工作重点. 另 外, 对于早三叠世氮代谢微生物功能群的变化特征 目前更不清楚.

需要指出的是, 这里所展示的生物对环境的作 用仅仅是冰山一角, 还有更多的生物对环境的作用 需要挖掘. 另外, 上述的讨论全部还只是停留在定性 的分析上, 缺乏定量/半定量的分析, 这也是今后研 究中的重点. 现代微生物对环境作用的深入认识以 及室内实验的定量/半定量分析, 是深入探讨地质历 史时期生物对环境作用的关键. 只有充分认识到生 物对环境的作用, 才能更好地探讨生物与环境的相 互作用及协同演化，进而对当代人与地球的可持续 发展提供启示.

\section{参考文献}

黄咸雨, 焦丹, 鲁立强, 等. 2007. 二叠纪-三叠纪之交环境的不稳定性和生物危机的多阶段性: 浙江长兴微生物分子化石记录. 中国科学 D 辑: 地球科学, 37: 629-635

罗根明. 2012. 二叠纪-三叠纪之交的微生物地质过程和 C-N-S 生物地球化学循环. 武汉: 中国地质大学博士学位论文, 1-20 孙枢, 王成善. 2008. Gaia 理论与地球系统科学. 地质学报, 82: 1-8

王永标, 童金南, 周修高, 等. 2005. 华南二叠纪末大灭绝后的钻质微生物岩及古环境意义. 科学通报, 50: 552-558

谢树成, 龚一鸣, 童金南, 等. 2006. 从古生物学到地球生物学的跨越. 科学通报, 51:2327-2336 
谢树成, 殷鸿福, 史小颖. 2011. 地球生物学: 生命与地球环境的相互作用和协同演化. 北京: 科学出版社

谢树成, 杨欢, 罗根明, 等. 2012. 地质微生物功能群: 生命与环境相互作用的重要突破口. 科学通报, 57: 3-22

殷鸿福, 谢树成, 秦建中, 等. 2008. 对地球生物学、生物地质学和地球生物相的一些探讨. 中国科学 D 辑: 地球科学, 38: 1473-1480

张元动, 詹仁斌, 㚞隽轩, 等. 2010. 奥陶纪生物大辐射研究的关键科学问题. 中国科学: 地球科学, 39: 129-1438

Algeo T J, Scheckler S E, Maynard J B. 2001. Effects of early vascular land plants on weathering processes and global chemical fluxes during the Middle and Late Devonian. In: Gensel P, Edwards D, eds. Plants Invade the Land: Evolutionary and Environmental Perspectives. Columbia: Columbia University Press. 213-236

Algeo T J, Hannigan R, Rowe H, et al. 2007. Sequencing events across the Permian-Triassic boundary, Guryul Ravine(Kashmir, India). Paleogeogr Paleoclimatol Paleoecol, 252: 328-346

Algeo T J, Shen Y, Zhang T, et al. 2008. Association of ${ }^{34}$ S-depleted pyrite layers with negative carbonate $\delta^{13} \mathrm{C}$ excursions at the Permian/Triassic boundary: Evidence for upwelling of sulfidic deep-ocean watermasses. Geochem Geophys Geosyst, 9: Q04025

Algeo T, Henderson C M, Ellwood B, et al. 2012. Evidence for a diachronous Late Permian marine crisis from the Canadian Arctic region. Geol Soc Am Bull, 124: 1424-1448

Anbar A D, Knoll A H. 2002. Proterozoic ocean chemistry and evolution: A bioinorganic bridge? Science, 297: 1137-1143

Baud A, Richoz S, Pruss S. 2007. The lower Triassic anachronistic carbonate facies in space and time. Glob Planet Change, 55: 81-89

Beaumont V, Robert F. 1999. Nitrogen isotoe ratios of kerogens in Precambrian cherts: A record of the evolution of atmosphere chemistry? Precambrian Res, 96: 63-82

Blankenship R. 2001. Molecular evidence for the evolution of photosynthesis. Trends Plant Sci, 6: 4-6

Blumenberg M, Mollenhauer G, Zabel M, et al. 2010. Decoupling of bio- and geohopanoids in sediments of the Benguela Upwelling System (BUS). Org Geochem, 41: 1119-1129

Borgonie G, Garcia-Moyano A, Litthauer D, et al. 2011. Nematoda from the terrestrial deep subsurface of South Africa. Nature, 474: 79-82

Brocks J J, Love G D, Summons R E, et al. 2005. Biomarker evidence for green and purple sulphur bacteria in a stratified Palaeoproterozoic sea. Nature, 437: 866-870

Buick R. 2007. Did the Proterozoic 'Canfield Ocean' cause a laughing gas greenhouse? Geobiology, 5: 97-100

Burhan R Y P, Trendel J M, Adam P, et al. 2002. Fossil bacterial ecosystem at methane seeps: Origin of organic matter from Be'eri sulfur deposit, Israel. Geochim Cosmochim Acta, 66: 4085-4101

Canfield D E. 1998. A new model for Proterozoic ocean chemistry. Nature, 396: 450-453

Canfield D E, Kristensen E, Thamdrup B. 2004. Aquatic Geomicrobiology. California: Elsevier Academic Press. 1-640

Canfield D E, Stewart F J, Thamdrup B, et al. 2010a. A cryptic sulfur cycle in oxygen-minimum-zone waters off the Chilean coast. Science, 330: 1375-1378

Canfield D E, Glazer A N, Falkowski P G. 2010b. The evolution and future of Earth's nitrogen cycle. Science, 330: 192-196

Cao C Q, Love G D, Hays L E, et al. 2009. Biogeochemical evidence for euxinic oceans and ecological disturbance presaging the end-Permian mass extinction event. Earth Planet Sci Lett, 281: 188-201

Capone D G, Zehr J P, Paerl H W, et al. 1997. Trichodesmium, a globally significant marine cyanobacterium. Science, 276: 1221-1229

Chen Z Q, Benton MJ, 2012. The timing and pattern of biotic recovery following the end-Permian mass extinction. Nat Geosci, 5: 375-383

Cvejic J H, Bodrossy L, Kovacs K L, et al. 2000. Bacterial triterpenoids of the hopane series from the methanotrophic bacteria Methylocaldum spp.: Phylogenetic implications and first evidence for an unsaturated aminobacteriohopanepolyol. Fems Microbiol Lett, 182: 361-365

Dannenberg S, Kroder M, Dilling W. 1992. Oxidation of $\mathrm{H}_{2}$, organic-compounds and inorganic sulfur-compounds coupled to reduction of $\mathrm{O}_{2}$ or nitrate by sulphate-reducing bacteria. Arch Microbiol, 158: 93-99

D’Hondt S, Jørgensen B B, Miller D J, et al. 2004. Distributions of microbial activities in deep subseafloor sediments. Science, 306: 2216-2221

Erwin D H. 2006. Extinction: How Life on Earth Nearly Ended 250 Million Years Ago. New York: Princeton University Press. 320

Falkowski P G. 1997. Evolution of the nitrogen cycle and its influence on the biological sequencestration of $\mathrm{CO}_{2}$ in the ocean. Nature, 387 : 272-275

Fareleira P, Santos B S, António C, et al. 2003. Response of a strict anaerobe to oxygen: Survival strategies in Desulfovibriogigas. Microbiology, 149: $1513-1522$

Farrimond P, Talbot H M, Watson D F, et al. 2004. Methylhopanoids: Molecular indicators of ancient bacteria and a petroleum correlation tool. Geochim Cosmochim Acta, 68: 3873-3882

Garvin J, Buick R, Anbar A D, et al. 2009. Isotopic evidence for an aerobic nitrogen cycle in the latest Archean. Science, 323: 1045-1048

Gold T. 1992. The deep, hot biosphere. Proc Natl Acad Sci USA, 89: 6045-6049 
Gorjan P, Kaiho K, Kakegawa T, et al. 2007. Paleoredox, biotic and sulfur-isotope changes associated with the End-Permian mass extinction in the western Tethys. Chem Geol, 244: 483-492

Grasby S E, Beauchamp B, Embry A, et al. 2012. Recurrent Early Triassic ocean anoxia. Geology, 41: 175-178

Grice K, Cao C Q, Love G D. 2005. Photic zone euxinia during the Permian-Triassic superanoxic event. Science, 307: 706-709

Gruber N, Galloway J N. 2008. An Earth-system perspective of the global nitrogen cycle. Nature, 451: 293-296

Haltia T, Brown K, Tegoni M, et al. 2003. Crystal structure of nitrous oxide reductase from paracoccus denitrificans at $1.6 \AA$ resolution. Biochem J, 369: 77-88

Hanson R S, Hanson T E. 1996. Methanotrophic Bacteria. Microbiol Rev, 60: 439-471

Harper D A T. 2006. The Ordovician biodiversification: Setting an agenda for marine life. Paleogeogr Paleoclimatol Paleoecol, 232: 148-166

Hays L E, Beatty T, Henderson C M, et al. 2007. Evidence for photic zone euxinia through the End-Permian mass extinction in the Panthalassic Ocean (Peace River Basin, Western Canada). Palaeoworld, 16: 39-50

Hays L E, Grice K, Foster C B, et al. 2012. Biomarker and isotopic trends in a Permian-Triassic sedimentary section at Kap Stosch, Greenland. Org Geochem, 43: 67-82

Hinrichs K U, Hayes J M, Sylva S P, et al. 1999. Methane-consuming archaebacteria in marine sediments. Nature, 398: 802-805

Hoehler T M, Alperin M J, Albert D B. 1998. Thermodynamic control on hydrogen concentrations in anoxic sediments. Geochim Cosmochim Acta, 62: 1745-1756

Hönisch B, Ridgwell A, Schmidt D N, et al. 2012. The geological record of ocean acidification. Science, 335: 1058-1063

Horita J, Zimmermann H, Holland H D. 2002. Chemical evolution of seawater during the Phanerozoic: Implications from the record of marine evaporites. Geochim Cosmochim Acta, 66: 3733-3756

Hsü K J, McKenzie J A. 1985. A "Strangelove" ocean in the earliest Tertiary. In: Sundquist E T, Broecker W S, eds. The Carbon Cycle and Atmospheric $\mathrm{CO}_{2}$ : Natural Variations Archean to Present. AGU Geophys Monogr, 32: 487-492

Jia C L, Huang J H, Kershaw S, et al. 2012. Microbial response to limited nutrients in shallow water immediately after the End-Permian mass extinction. Geobiology, 10: 60-71

Joachimski M M, Lai X L, Shen S Z, et al. 2012. Climate warming in the latest Permian and the Permian-Triassic mass extinction. Geology, 40: 195-198

Jørgensen B B. 1982. Mineralization of organic matter in the sea bed-the role of sulphate reduction. Nature, 296: 643-645

Jørgensen B B, Kasten S. 2006. Sulfur cycling and methane oxidation. In: Schulz H D, Zabel M, eds. Marine Geochemistry. London: Springer. 271-309

Kaiho K, Kajiwara Y, Nakano T, et al. 2001. End Permian catastrophe by a bolide impact: Evidence of a gigantic release of sulfur from the mantle. Geology, 29: 815-818

Kaiho K, Kajiwara Y, Chen Z Q, et al. 2006. A sulfur isotope event at the end of the Permian. Chem Geol, 235: 33-47

Kasting J F. 2005. Methane and climate during the Precambrian era. Precambrian Res, 137: 119-129

Khalil M A K. 1999. Non- $\mathrm{CO}_{2}$ greenhouse gases in the atmosphere. Annu Rev Energ Env, 24: 645-661

King G M, Roslev P, Skovgaard H. 1990. Distribution and rate of methane oxidation in sediments of the Florida Everglades. Appl Environ Microbiol, 56: 2902-2911

Knoll A H, Bambach R K, Payne J L, et al. 2007. Paleophysiology and end-Permian mass extinction. Earth Planet Sci Lett, 256: 295-313

Konhauser K. 2007. Introduction to Geomicrobiology. Malden: Blackwell Publishing. 1-405

Kump L R, Pavlov A, Arthur M A. 2005. Massive release of hydrogen sulfide to the surface ocean and atmosphere during interval of oceanic anoxia. Geology, 33: 397-400

Lavik G, Stührmann T, Brüchert V, et al. 2009. Detoxification of sulphidic African shelf waters by blooming chemolithotrophs. Nature, 457: 581-584

Lehrmann D J, Payne J L, Felix S V, et al. 2003. Permian-Triassic boundary sections from shallow-marine carbonate platforms of the NanpanjiangBasin, South China: Implications for oceanic conditions associated with the End-Permian extinction and its aftermath. Palaios, 18: 138-152

Lemos R S, Gomes C M, Santana M. 2001. The "strict" anaerobe Desulfovibriogigas contains a membrane-bound oxygen respiratory chain. Febs Lett, 496: 40-43

Lenton T M, Schellnhuber H J, Szathmary E. 2004. Climbing the co-evolution ladder. Nature, 431: 913

Li F, Yan J, Algeo T, et al. 2013. Paleoceanographic conditions following the End-Permian mass extinction recorded by giant ooids (Moyang, South China). Glob Planet Change, 105: 102-120 
Liao W, Wang Y B, Kershaw S, et al. 2010. Shallow-marine dysoxia across the Permian-Triassic boundary: Evidence from pyrite framboids in the microbialite in South China. Sediment Geol, 232: 77-83

Lipp J S, Morono Y, Inagaki F, et al. 2008. Significant contribution of Archaea to extant biomass in marine subsurface sediments. Nature, 454: 991-994

Lovelock J E, 1972. Gaia as seen through the atmosphere. Atmos Environ, 6: 452-453

Lowenstein T K, Hardie L A, Timofeeff M N, et al. 2003. Secular variation in seawater chemistry and the origin of calcium chloride basinal brines. Geology, 31: 857-860

Lowenstein T K, Timofeeff M N, Kovalevych V M, et al. 2005. The major-ion composition of Permian seawater. Geochim Cosmochim Acta, 69: $1701-1719$

Luo G M, Kump L R, Wang Y B, et al. 2010. Isotopic evidence for an anomalously low oceanic sulphate concentration following End-Permian mass extinction. Earth Planet Sci Lett, 300: 101-111

Luo G M, Wang Y B, Algeo T J, et al. 2011. Enhanced nitrogen fixation in the immediate aftermath of the latest Permian marine mass extinction. Geology, 39: 647-650

Luo G M, Wang Y B, Grice K, et al. 2013. Microbial-algal community changes during the latest Permian ecological crisis: Evidence from lipid biomarkers at Cili, South China. Glob Planet Change, 105: 36-51

Moisander P H, Beinart R A, Hewson I, et al. 2010. Unicellular cyanobacterial distributions broaden the oceanic $\mathrm{N}_{2}$ fixation domain. Science, 327 : $1512-1514$

Mumma M J, Villanueva G L, Novak R E, et al. 2009. Strong release of methane on Mars in Northern Summer 2003. Science, 323: 1041-1045

Nabbefeld B, Grice K, Twitchett R J, et al. 2010. An integrated biomarker, isotopic and palaeoenvironmental study through the Late Permian event at Lusitaniadalen, Spitsbergen. Earth Planet Sci Lett, 291: 84-96

Naqvi S W A, Yoshinari T, Jayakumar D A, et al. 1998. Budgetary and biogeochemical implications of $\mathrm{N}_{2} \mathrm{O}$ isotope signatures in the Arabian Sea. Nature, 394: 462-464

Navarro-Gonzalez R, McKay C P, Mvondo D N. 2001. A possible nitrogen crisis for Archaean life due to reduced nitrogen fixation by lightning. Nature, 412: 61-64

Newton R J, Pevitt E L, Wignall P B, et al. 2004. Large shifts in the isotopic composition of seawater sulphate across the Permian-Triassic boundary in northern Italy. Earth Planet Sci Lett, 218: 331-345

Orphan V J, Hinrichs K U, Ussler III W, et al. 2001. Comparative analysis of methane-oxidizing archaea and sulfate-reducing bacteria in anoxic marine sediments. Appl Environ Microbiol, 67: 1922-1934

Parkes R J, Cragg B A, Bale S J, et al. 1994. Deep bacterial biosphere in Pacific Ocean sediments. Nature, 371: 410-413

Peckmann J, Thiel V, Michaelis W, et al. 1999. Cold seep deposits of Beauvoisin (Oxfordian; southeastern France) and Marmorito (Miocene; northern Italy): Microbially induced authigenic carbonates. Int J Earth Sci, 88: 60-75

Pedersen K. 1993. The deep subterranean biosphere. Earth-Sci Rev, 34: 243-260

Peters K E, Walters C C, Moldowan J M. 2005. The Biomarker Guide: Biomarkers and isotopes in the environment and human history. Cambridge: Cambridge University Press. 704

Rabus A, Hansen T A, Widdel F. 2006. Dissimilatory sulfate- and sulfur-reducing prokaryotes. In: Dworkin M, Falkow S, Rosenberg E, et al. eds. The Prokaryotes. New York: Springer Science+Business Media. 2: 659-768

Riccardi A L, Arthur M A, Kump L R. 2006. Sulfur isotopic evidence for chemocline upward excursions during the End-Permian mass extinction. Geochim Cosmochim Acta, 70: 5740-5752

Romano, C, Goudemand, N, Vennemann, T W, et al. 2012. Climatic and biotic upheavals following the End-Permian mass extinction. Nat Geosci, 6: $57-60$

Saito M A, Sigman D M, Morel F M M. 2003. The bioinorganic chemistry of the ancient ocean: The co-evolution of cyanobacterial metal requirements and biogeochemical cycles at the Archean-Proterozoic boundary? Inorg Chim Acta, 356: 308-318

Saito R, Oba M, Kaiho K, et al. 2013. Ether lipids from the Lower and Middle Triassic at Qingyan, GuizhouProvince, Southern China. Org Geochem, 58: 27-42

Schoepfer S D, Henderson C M, Garrison G H, et al. 2012. Cessation of a productive coastal upwelling system in the PanthalassicOcean at the Permian-Triassic Boundary. Paleogeogr Paleoclimatol Paleoecol, 313-314: 181-188

Scholten J C M, van Bodegom P M, Vogelaar J, et al. 2002. Effect of sulfate and nitrate on acetate conversion by anaerobic microorganisms in a freshwater sediment. Fems Microbiol Ecol, 42: 375-385

Severin I, Stal L J. 2008. Light dependency of nitrogen fixation in a coastal cyanobacterial mat. ISME J, 2: 1077-1088 
Severin I, Stal L J. 2010. Diazotrophic microbial mats. In: Seckbach J, Oren A, eds. Microbial Mats: Modern and Ancient Microorganisms in Stratified Systems, Cellular Origin, Life in Extreme Habitats and Astrobiology. New York: Springer Science+Business Media. 14: 321-339

Shen S Z, Crowley J L, Wang Y, et al. 2011. Calibrating the End-Permian mass extinction. Science, 334: 1367-1372

Shen W, Lin Y, Xu L, et al. 2007. Pyrite framboids in the Permian-Triassic boundary section at Meishan, China: Evidence for dysoxic deposition. Paleogeogr Paleoclimatol Paleoecol, 253: 323-331

Shen Y A, Buick R, Canfield D E. 2001. Isotopic evidence for microbial sulphate reduction in the early Archaean era. Nature, 410: 77-81

Sinninghe Damsté J S, Köster J. 1998. A euxinic southern North Atlantic Ocean during the Cenomanian/Turonian oceanic anoxic event. Earth Planet Sci Lett, 158: 165-173

Sohm J A, Webb E A, Capone D G. 2011. Emerging patterns of marine nitrogen fixation. Nat Rev Microbiol, 9: 499-508

Song H J, Wignall P, Tong J N, et al. 2012. Geochemical evidence from bio-apatite for multiple oceanic anoxic events during Permian-Triassic transition and the link with End-Permian extinction and recovery. Earth Planet Sci Lett, 253-254: 12-21

Song H Y, Tong J, Algeo T J, et al. 2014. Concurrent changes in the marine sulfur and carbon cycles during the Early Triassic: Evidence for ocean stagnation and subsequent overturn. Geochim Cosmochim Acta, in press

Summons R E, Powell T G. 1987. Identification of aryl isoprenoids in source rocks and crude oils: Biological markers for the green sulphur bacteria. Geochim Cosmochim Acta, 51: 557-566

Sun Y D, Joachimski M M, Wignall P B, et al. 2012. Lethally hot temperatures during the Early Triassic greenhouse. Science, 338: 366-370

Thauer R K, Stackebrandt E, Hamilton W A. 2006. Energy metabolism and phylogenetic diversity of sulphate-reducing bacteria. In: Barton L L, Hamilton W A, eds. Sulphate-reducing Bacteria: Environmental and Engineered Systems. Cambridge: CambridgeUniversity Press. 1-37

Tyrrell T. 1999. The relative influences of nitrogen and phosphorus on oceanic primary production. Nature, 400: 525-531

Ueno Y, Yamada K, Yoshida N, et al. 2006. Evidence from fluid inclusions for microbial methanogenesis in the early Archaean era. Nature, 440: 516-519

Valentine D L. 2002. Biogeochemistry and microbial ecology of methane oxidation in anoxic environments: Areview. Antonie van Leeuwenhoek, 81: 271-282

van Breugel Y, Schouten S, Paetzel M, et al. 2005. The impact of recycling of organic carbon on the stable carbon isotopic composition of dissolved inorganic carbon in a stratified marine system (Kyllaren fjord, Norway). Org Geochem, 36: 1163-1173

Wacey D, McLoughlin N, Whitehouse M J, et al. 2010. Two coexisting sulfur metabolisms in a ca. 3400 Ma sandstone. Geology, 38: 1115-1118

Ward P D, Botha J, Buick R, et al. 2005. Abrupt and gradual extinction among Late Permian land vertebrates in the Karoo Basin, South Africa. Science, 307: 709-715

Webby B D, Paris F, Droser M L, et al. 2004. The Great Ordovician Biodiversification Event. New York: Columbia University Press. 1-487

Whitman W B, Coleman D C, Wiebe W J. 1998. Prokaryotes: The unseen majority. Proc Natl Acad Sci USA, 95: 6578-6583

Wignall P B, Newton R, Brookfield M E. 2005. Pyrite framboid evidence for oxygen-poor deposition during the Permian-Triassic crisis in Kashmir. Paleogeogr Paleoclimatol Paleoecol, 216: 183-188

Wignall P B, Bond D P G, Kuwahara K, et al. 2010. An 80 million year oceanic redox history from Permian to Jurassic pelagic sediments of the Mino-Tamba terrane, SW Japan, and the origin of four mass extinctions. Glob Planet Change, 71: 109-123

Wilkin R T, Barnes H L. 1997. Formation processes of framboidal pyrite. Geochim Cosmochim Acta, 61: 323-339

Xie S C, Pancost R D, Yin H F, et al. 2005. Two episodes of microbial change coupled with Permo/Triassic faunal mass extinction. Nature, 434: 494-497

Yin H F, Feng Q L, Lai X L, et al. 2007. The protracted Permo-Triassic crisis and the multi-episode mass extinction around the Permian-Triassic boundary. Glob Planet Change, 55: 1-20

Yin H F, Xie S C, Luo G M, et al. 2012. Two episodes of environmental change at the Permian-Triassic boundary of the GSSP section Meishan. Earth-Sci Rev, 115: 163-172

Zehr J P, Mellon M, Braun S, et al. 1995. Diversity of heterotrophic nitrogen fixation genes in a marine cyanobacterial mat. Appl Environ Microbiol, 61: 2527-2532

Zehr J P, Waterbury J B, Turner P J, et al. 2001. Unicellular cyanobacteria fix $\mathrm{N}_{2}$ in the subtropical North Pacific Ocean. Nature, 412: 635-638

Zehr J P, Bench S R, Carter B J, et al. 2008. Globally distributed uncultivated oceanic $\mathrm{N}_{2}$-fixing cyanobacteria lack oxyenic photosystem II. Science, 322: 1110-1112

Zerkle A L, Kamyshny Jr A, Kump L R, et al. 2010. Sulfur cycling in a stratified euxinic lake with moderately high sulfate: Constraints from quadruple S isotopes. Geochim Cosmochim Acta, 74: 4953-4970 